\title{
Dissipative Kerr solitons and microcavity frequency combs (Conference Presentation)
}

Kerry J. Vahala, California Institute of Technology (United States); Vladimir S. Ilchenko, OEwaves, Inc. (United States)

\begin{abstract}
Dissipative Kerr soliton mode locking in high-Q silica micro cavities is reviewed including resonator dispersion optimization. Phenomena relating to soliton propagation in the micro cavity are studied including dispersive wave generation and soliton trapping. Applications of the soliton comb are described.

View presentation recording on the SPIE Digital Library:

http://dx.doi.org/10.1117/12.2255667.5387835764001
\end{abstract}

